\title{
Machine learning: Great opportunities, but will it replace nurses?
}

The beginning of a New Year is a good time to look forward to the brave new world we are moving into, in which computer-based technologies are increasingly common. We hear a great deal about the advances being made in the development of artificial intelligence (AI) and machine learning (ML). Similarly often, we are warned that Al will not only transform the workplace but lead to loss of jobs and possibly whole professions. What are the implications and the opportunities for nursing?

As a field of study, Al has been around for more than half a century. Increasingly sophisticated computational technologies use data from multiple sources including sensors to modify algorithms to inform activity, using rules to establish learning, and reasoning processes. With increasing computer power, more recently $\mathrm{ML}$ has expanded what can be "learnt," using statistical techniques analysing big datasets. The results are appearing in the market place, in industry, and in our everyday lives. Increasingly we use, for example, Al in video gaming, speech-based "apps" on our phones and within our homes and apply as safety features functions, which will in the future enable cars to be self-driving (Stone et al., 2016).

We hear about the opportunities of $\mathrm{ML}$ in clinical practice. Most often, these relate to faster, cheaper diagnostics, and may mean services can be offered where the required expert resource would not otherwise be accessible or financially viable. In medical imaging, for example, ML may revolutionize identification of clinical signs, using pattern recognition to predict likely diagnosis or referrals. Evaluation is showing performance accuracy equaling human experts but at much faster speeds (De Fauw et al., 2018; Rajpurkar et al., 2018). As well as automating stages of diagnosis, ML may expand our current definitions of diseases and their subtypes. For example, rather than the traditional four main diabetes subgroups (types 1 and 2, LADA, and gestational), recent computational work has described a further five subgroups. Ahlqvist et al (2018) link lack of recognition of this to current suboptimal treatment. To date nursing diagnoses have not been subjects of similar work.

Patient safety and quality are another area where ML may automate procedures. The mapping of care processes and identification of errors often entails chart reviews, rule-based screening of electronic medical records, and significant event audits. Such procedures, often carried out by nurses, are labour intensive and can be error prone, as much due to faults in recording as extraction of the data. $M L$ techniques can model what is expected based on historical data and use these models to flag "outlier" events, occurring at low probability. Using a similar process to identify medication errors, Schiff et al (2017) reported that three quarters of the alerts generated were accurate reflections of the healthcare record, and three quarters of the valid alerts were clinically useful. However, as long as health records include manually entered data, this approach is unlikely to ever replace human input but may usefully reduce what is often nursing workload.

As well as automating existing workflows, ML may augment them. For example, a software program using routinely collected data was able to identify patients at high risk of post-surgical complications or death, with one in three patients flagged by the software experiencing an event within 30 days. This facilitated individualization of preventive care (Corey et al., 2018). Similar approaches are being applied in other areas, including sepsis pathways (Henry, Wongvibulsin, Zhan, Saria, \& Hager, 2017) and prediction of unplanned readmissions (Health Catalyst, 2018). Potential future nursing applications might include areas such as Emergency Dept triage.

Serving similar augmentive functions, examples are multiplying of human-like robot nurses, "nursebots," being used for support functions. In Japan, for example, they carry out tasks such as moving, bathing, and dressing elderly patients and can conduct simple conversations. In many countries, bots are serving hospitality-type functions (greeting, providing information, and way finding). Similar functions have been embedded with a virtual nurse in home monitoring systems for patients with chronic disease, providing information in response to patient queries. Whilst for some, this represents a "thin end of the wedge" threat for nursing, once again $M L$ is providing additive rather than replacement nursing functions, with chatbots using sophisticated natural language processing (De Jesus, 2018). However, extension of these functions into areas where decisions are taken for individual patients is tempered by the limitations of current system development processes.

To date ML algorithms are fed by routinely available datasets, which only provide partial models. For example, consider emergency admissions: prediction of future emergency admissions based on previous patterns of admissions may be adequately prognostic for service planning yet useless for individual patients as algorithms cannot currently accommodate influential factors such as the decisional habits of individual admitting doctors, bed availability, or patients' health insurance status. Training datasets may also contain patterns unique to that data subset rather than the source population, resulting in "overfitting" or spurious associations (Saria, Butte, \& Sheikh, 2018).

These are challenges for the future and illustrate why it is essential that, rather than being distracted by media scare stories, nurses, and all healthcare professional groups need to understand what ML can offer and work with technology developers to leverage it to improve patient care and appropriately complement nursing workflows. 


\section{ORCID}

Lin Perry (D) https://orcid.org/0000-0002-8507-1283

\author{
Lin Perry PhD, MSc, RN iD \\ Professor, Editor-in-Chief \\ International Journal of Nursing Practice
}

\section{REFERENCES}

Ahlqvist, E., Storm, P., Karajamaki, A., Martinell, M., Dorkhan, M., Carlsson, A., ... Wessman, Y. (2018). Novel subgroups of adult-onset diabetes and their association with outcomes: A data-driven cluster analysis of six variables. The Lancet Diabetes \& Endocrinology, 6(5), 361-369. https://doi.org/10.1016/S2213-8587(18)30051-2

Corey, K. M., Kashyap, S., Lorenzi, E., Lagoo-Deenadayalan, S. A., Heller, K., Whalen, K., ... Sendak, M. (2018). Development and validation of machine learning models to identify high-risk surgical patients using automatically curated electronic health record data (Pythia): A retrospective, single-site study. PLoS Medicine, 15(11), e1002701. https:// doi.org/10.1371/journal.pmed.1002701. 30481172

De Fauw, J., Ledsam, J. R., Romera-Paredes, B., Nikolov, S., Tomasev, N., Blackwell, S., ... van den Driessche, G. (2018). Clinically applicable deep learning for diagnosis and referral in retinal disease. Nature Medicine, 24(9), 1342-1350. https://doi.org/10.1038/s41591-018-0107-6

De Jesus, A. (2018). Machine learning for nursing-8 current applications. Automation and Robotics: Healthcare 2018. Retrieved from https:// emerj.com/ai-sector-overviews/machine-learning-for-nursing-8-current-applications/

Health Catalyst (2018). Retrieved from https://emerj.com/ai-sector-overviews/machine-learning-for-nursing-8-current-applications/

Henry, K., Wongvibulsin, S., Zhan, A., Saria, S., \& Hager, D. (2017). Can septic shock be identified early? Evaluating performance of A targeted real-time early warning score (TREWScore) for septic shock in a community hospital: Global and subpopulation performance. American Journal of Respiratory and Critical Care Medicine, 195, A7016.

Rajpurkar, P., Irvin, J., Ball, R. L., Zhu, K., Yang, B., Mehta, H., ... Lungren, M. P. (2018). Deep learning for chest radiograph diagnosis: A retrospective comparison of the CheXNeXt algorithm to practicing radiologists. PLoS Medicine, 15(11), e1002686. https://doi.org/10.1371/journal. pmed.1002686

Saria, S., Butte, A., \& Sheikh, A. (2018). Better medicine through machine learning: What's real, and what's artificial? PLoS Medicine, 15(12), e1002721. https://doi.org/10.1371/journal.pmed.1002721

Schiff, G. D., Volk, L. A., Volodarskaya, M., Williams, D. H., Walsh, L., Myers, S. G., ... Rozenblum, R. (2017). Screening for medication errors using an outlier detection system. Journal of the American Medical Informatics Association, 24(2), 281-287. https://doi.org/10.1093/jamia/ ocw171

Stone, P., Brooks, R., Brynjolfsson, E., Calo, R., Etzioni, O., Hager, G., ... Teller, A. (2016). Artificial intelligence and life in 2030. One hundred year study on artificial intelligence: Report of the 2015-2016 study panel. Stanford, CA: Stanford University. Retrieved from http://ai100. stanford.edu/2016-report. 